The $8^{\text {th }}$ International Conference of the Slovenian Society for Non-Destructive Testing

"Application of Contemporary Non-Destructive Testing in Engineering"

September 1-3, 2005, Portorož, Slovenia, pp. 35-43

\title{
DEFECT DETECTION IN THIN PLATES BY ULTRASONIC LAMB WAVE TECHNIQUES
}

\author{
K. Edalati, A. Kermani, M. Seiedi, M. Movafeghi \\ Department of NDT, Nuclear Safety and Radiation Protection Technological Center of Iran, AEOI, North Kargar \\ Street, P.O. Box: 14155-1399, Tehran, Iran, E-mail: ndt99@aeoi.org.ir
}

\begin{abstract}
In the present study, the numerical method was investigated for drawing the dispersion and displacement curves of ultrasonic lamb wave, propagated in Aluminum thin plate. Two ultrasonic lamb wave techniques, pulse-echo (a1 mode emitter) and emission (s1 mode emitter), were used for interpretation of notch defects with depths of 10\%, 30\% and 60\% of plate thickness. Thickness of plate was $2 \mathrm{~mm}$ and the nominal center frequency of transducers was 2 $\mathrm{MHz}$. It was observed that these techniques are sensitive to evaluate defects, especially in short probe to defect distances. Also, it was found that amplitude analysis can give some qualitative information about defects depth by using Distance-Amplitude-Correction curves, but it was not sometime repeatable. In this respect, pulse echo method showed better sensitivity of amplitude to defects depth variation.
\end{abstract}

Keywords: Lamb wave, Thin plates, Displacement, Dispersion

\section{Introduction}

Ultrasonic lamb wave are being used for interpretation of plates [1], multilayer structures [2], pipes[3], rails [4], elbows [5], honeycomb structures [6], composite plates [2], and so on; also for materials characterization [7] (mainly elastic modules and stiffness constants). It can be used successfully for insulated [8] and coated pipes [9] as well as for inspection of objects under the water [10]. Lamb wave is a special kind of guided wave which can be travel over a long distance. The sensitivity of lamb wave to defects is greater than common ultrasonic method [11]. Lamb wave inspection is fast and its cost is so lower than common ultrasonic techniques or other inspection methods [3,10,11]. Angle probes, electromagnetic acoustic transducers [11], comb probe [12], immersion method [13] and laser ultrasonic technique [14] may be used for ultrasonic lamb wave testing. The most difficulty of lamb wave inspection is the dispersive nature of lamb wave and its multimode propagation and so need to signal processing as well as experienced operators [11,15]. Time-frequency analysis using Wavelet transform [15], Fourier transform and Fast Fourier transform [16] may be used for signal processing when different lamb modes were overlapped. The aim of present study is to evaluate defects in thin Aluminum plates. For this aim two lamb wave testing techniques (pulse-echo and emission), with different lamb modes as transmitter and receiver, have been used and the sensitivity of these methods has been compared with each other. 


\section{Theory}

The ultrasonic waves propagate in two main kinds in materials:

1- Longitudinal ultrasonic wave (L): displacement of particles is parallel with the energy transport direction,

2- Shear ultrasonic wave (S): displacement occurs in the direction perpendicular to energy transport.

Shear wave, as shown in Figure 1, can propagate in two main modes:

a- Shear horizontal wave (SH): displacement describes in the direction parallel to plate surface;

b- Shear vertical wave (SV): displacement occurs in the direction perpendicular to the plate surface.

In a free solid elastic thin plate (up to a few wavelengths of bulk ultrasonic wave thick), longitudinal wave are polarized in the direction perpendicular to the surface and SV wave formed and combined with $\mathrm{L}$ wave. These combined $\mathrm{SV}+\mathrm{L}$ is known as lamb wave [17]. In an isotropic plate, lamb waves are either symmetric $(\mathrm{Si})$ or antisymmetric $\left(\mathrm{a}_{\mathrm{i}}\right)$. The symmetric modes associate with a compressional rarefaction expansion of the plate and antisymmetric modes associated with a flexural propagation $[11,17]$. Lamb wave have a dispersive nature and its phase and group velocities not only depend on the material characterization but also depend on the thickness, frequency and lamb wave mode.

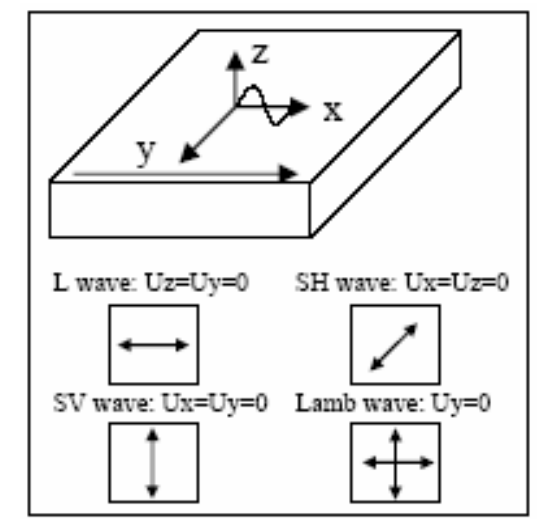

Fig. 1: Schematic illustration of ultrasonic wave modes.
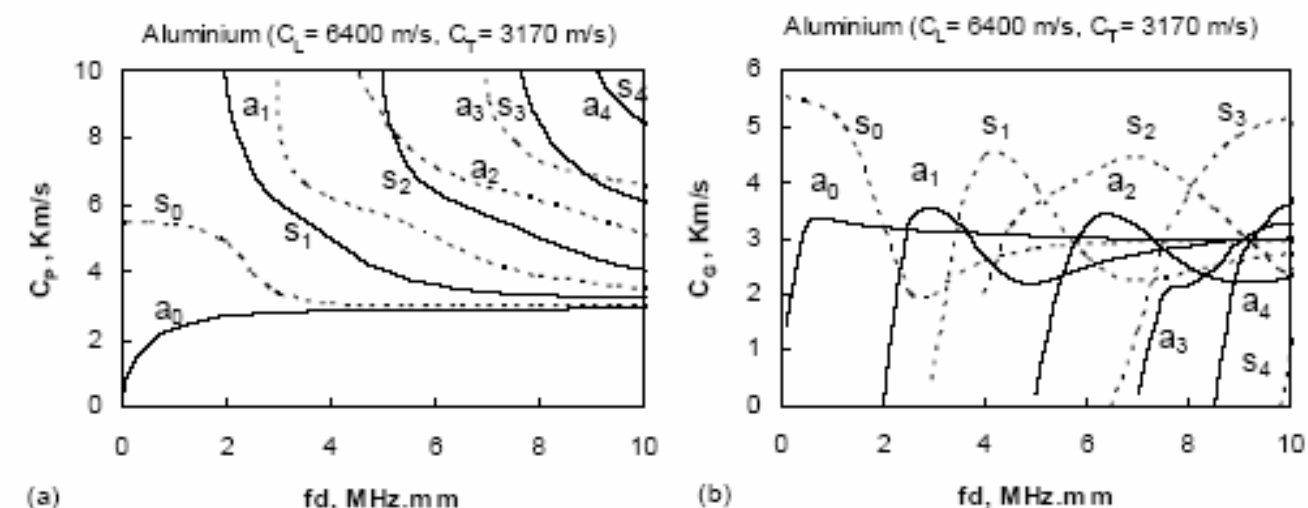

(b)

fd, $\mathrm{MHz} \cdot \mathrm{mm}$

Fig. 2: Lamb wave a) phase and b) group velocity dispersion curves, propagated in Aluminium plates.

When normal and shear stresses in the surface of plate reach to zero, then lamb wave can propagate $[11,17]$. The potential method is the most common approach for solving the lamb 
wave problems $[18,19]$. The effect of thickness, frequency and different modes of lamb wave on the phase and group velocity is defined in dispersion curves. For a single layer plate, the dispersion curves can be plotted by assessment the general dispersion equation, in a manner as defined in Appendix 1. The dispersion curves for an Aluminium plate are shown in Figures 2a and $2 b$.

It can be seen that at least two modes of lamb wave can propagate in a plate. Also lamb modes, except to a and so, have a cutoff frequency. So, for producing a special lamb mode the frequency should be higher than its cutoff frequency and via versa.

For a successful lamb wave testing, it is necessary to draw the dispersion curves. Any point on dispersion curves can be used for ultrasonic testing, but any mode of lamb wave is more sensitive to a special defect type [11]. Some modes are sensitive to coating or surface defects and some modes are sensitive to internal defects, and so on. Displacement curves can explain the sensitivity of lamb modes to defect types [9]. Incident angle and frequency are two parameters which can be varied for excitation a desired lamb wave testing. In the most researches, the frequency has been set for excitation two or three modes, which causing lower dispersion and easier inspection. Snell's law can be used for calculating the incident angle of the media between the transducer and plate for producing a special lamb mode with a defined phase velocity as is followed:

$$
\frac{C_{L m}}{C_{p}}=\sin \theta_{m}
$$

where $C_{L m}$ is the velocity of longitudinal ultrasonic wave in the separating media, $C_{P}$ is phase velocity of lamb wave mode and $\theta_{\mathrm{m}}$ is the incident angle in the separating media. The incident angle in water for immersion testing and in Perspex for variable angle probe has been shown in Figures $3 a$ and $3 b$.

In multilayer materials as compared with a single layer material, further modes propagate and these modes are neither symmetric nor antisymmetric [18,19]. Plotting the dispersion and displacement curves is also necessary for employing a successful lamb wave testing in multilayer plates. The transfer matrix methods may be applied for drawing the displacement profile of particles in plate when lamb wave propagate [20]. The dispersion curves in this method can be calculated in the manner as defined by Pan [9,21]. The displacement curves which are obtained by this method have been shown in Figures $4 \mathrm{a}$ and $4 \mathrm{~b}$. The mode of Figure $4 \mathrm{a}$ is interested for interpretation of internal defects of plate and the mode of Figure $4 \mathrm{~b}$ is desired for evaluation of discontinuities and delaminating in the interface of two layers.
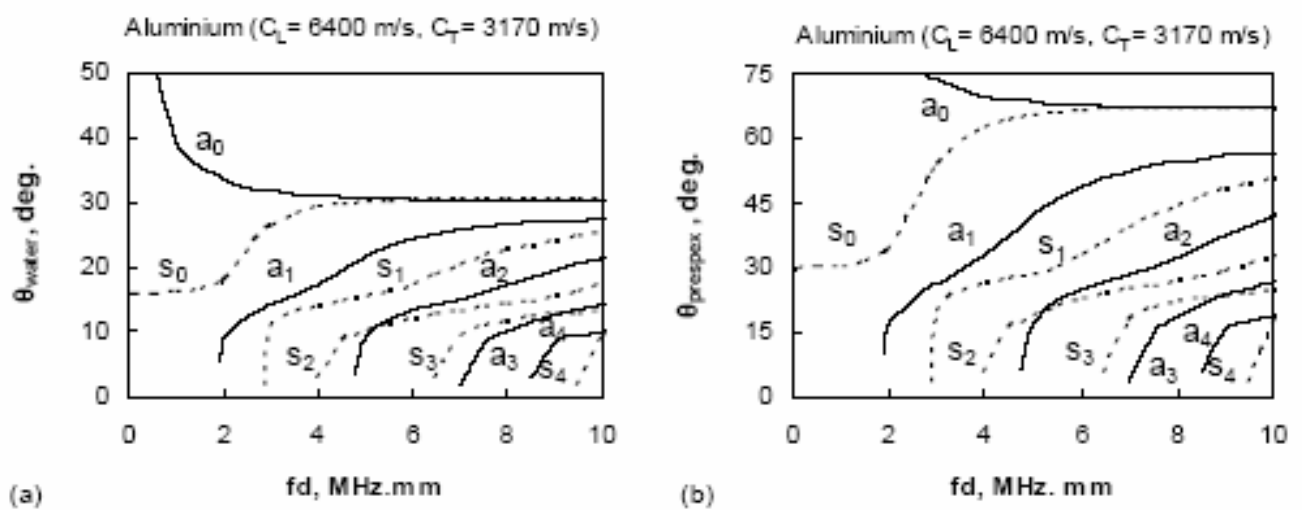

Fig. 3: The variations of incident angle in a) Water and b) Perspex for producing different lamb wave modes in Aluminium plates. 


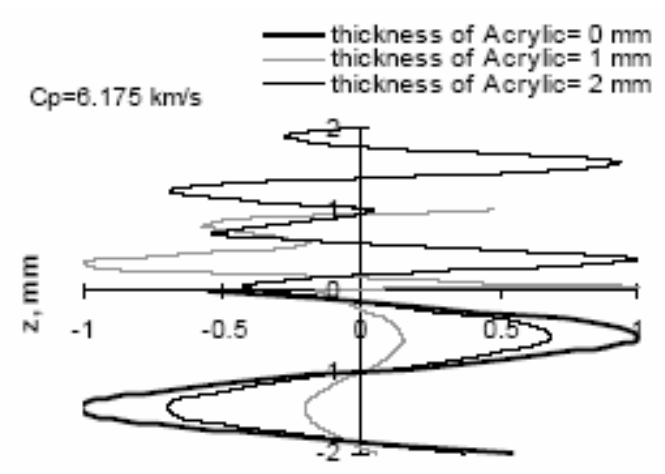

(a)

Normalized out-of-plane displacement

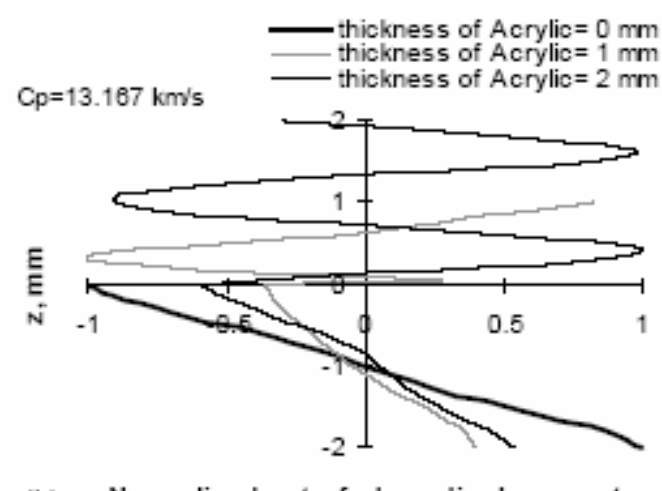

(b) Normalized out-of-plane displacement

Fig. 4: Out-of-plane displacement for an Aluminium plate laminated by Acrylic layers with thickness of 0,1 and $2 \mathrm{~mm}$.

\section{Experimental Procedures}

For the experimental trials a Aluminium plate, having $2 \mathrm{~mm}$ thickness, with specifications as shown in Figure 5, were provided. Plate was performed with notch defects, with $0.5 \mathrm{~mm}$ width and $10 \%, 30 \%$ and $60 \%$ of through wall depth.

Immersion test was used for measuring the real phase and group velocity of different modes of lamb wave propagated in plate. By changing the incident angle in water some peaks were appeared which some of them related to longitudinal wave propagation in water and others referred to ultrasonic lamb wave modes ( $\mathrm{s} 1$ mode appeared in $14^{\circ}$ and a1 mode appeared in $17^{\circ}$ and $\mathrm{a} 0$ and $\mathrm{s} 0$ overlapped in $30^{\circ}$ ). In most studies $\mathrm{s} 0$ and a0 modes have been applied for studies, but in the present work, a1 and si modes have been used for evaluation.

Two techniques have been applied for inspections: 1) pulse-echo by considering the a1 mode and using a variable angle probe, as shown in Fig. 6a and 2) emission method with s1 mode as transmitter and receiver and using two normal probe, as shown in Fig. 6b. Emission method is similar that pitch-catch technique but two normal probes are applied instead of angle probes. Emitted normal wave causes guided wave propagation when thickness of plate is enough small, due to resonance effect and polarisation of bulk wave in the top and bottom surfaces of plate.

In the present investigation, some experiments were made for studying the ability of these methods for interpretation of notch defects, having different depths. A 5 cycle of sine wave was excited at the $2 \mathrm{MHz}$ was used for examinations. The nominal center frequency of normal and angle transducers was $2 \mathrm{MHz}$. A fast Fourier transform was used for determining the real frequency of received signal, as shown as a sample in Fig. 7.

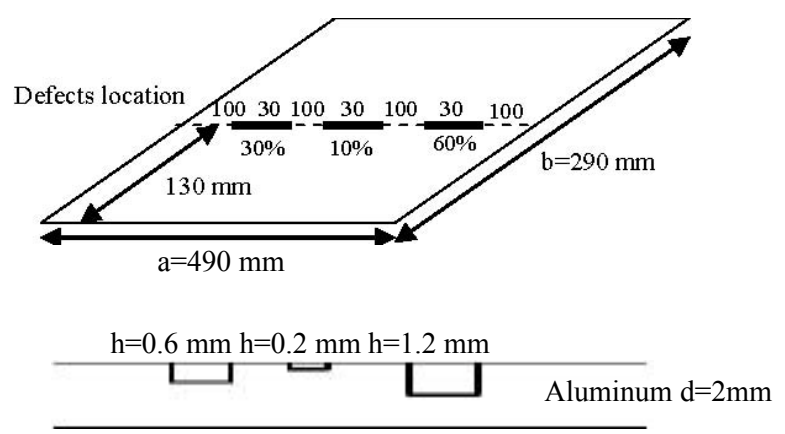

Fig. 5: The schematic mapping of fabricated aluminum plate. 

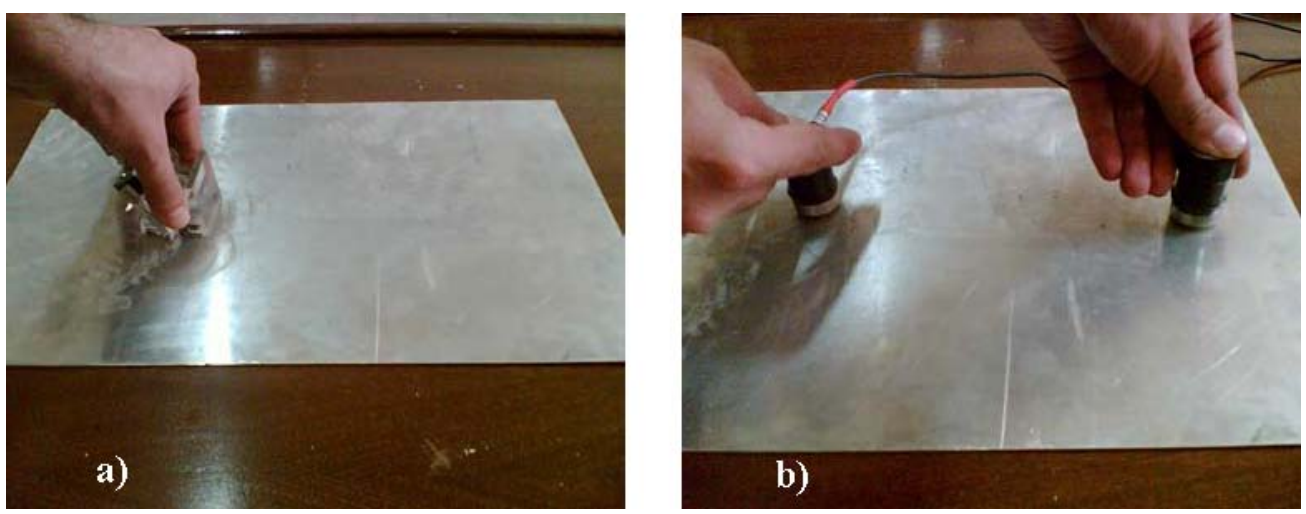

Fig. 6: The lamb wave testing methods: a) pulse-echo technique and b) emission method.
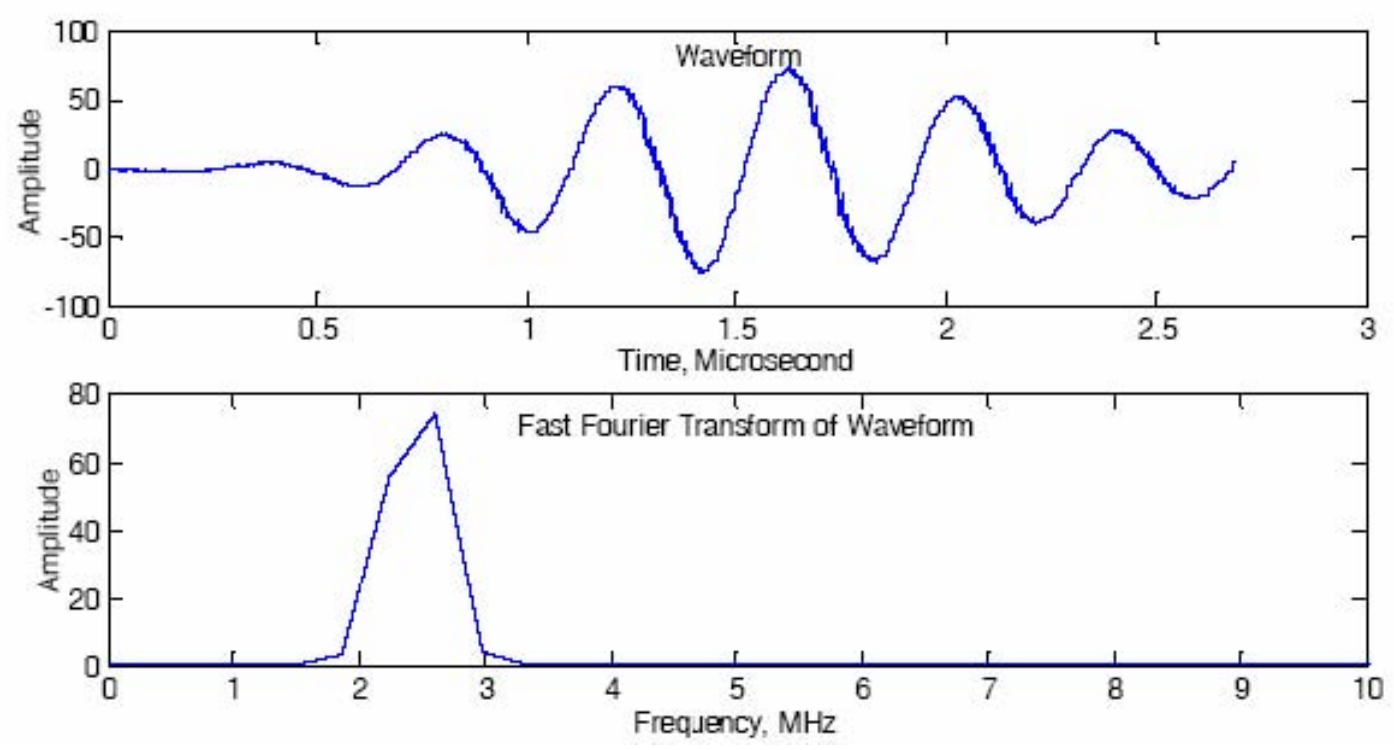

Fig. 7: A received waveform and its fast Fourier transform, showing the peak of frequency at $2.6 \mathrm{MHz}$.

\section{Results and Discussions}

The amplitude values which obtained by scanning the width of plate in a pulse-echo method have been shown in Figure 8. There is a powerful backwall peak in the flawless areas. When probe reach near the defect, amplitude of backwall peak decreases, due to beam spread. In defected zone, backwall peak become weak and a second peak appears. Second peak relate to reflection from defects. When defect depth increases, the reflecting surface increases, so, as seen in Figure 9, back wall peak will be weaker and defect peak will be stronger.

Time of flight and group velocity can be used for determining the location of defect. Group velocity may be calculated by dispersion curves or by assessment the backwall peak and self calibration (if the back wall reflection exists) as is followed:

$$
L d=V_{G} t_{o f}=\frac{L b}{m_{b}} m_{d}
$$


where $V_{G}$ is group velocity of lamb mode, $t_{o f}$ is time of flight of signal related to defect, $L_{d}$ and $\mathrm{L}_{\mathrm{b}}$ are respectively the location of defect and backwall and md and mb are the location of defect and backwall peaks on the time base axis of ultrasonic flaw detector screen. The results of measuring the defect and backwall location, measured by assessment the theoretical group velocity, are shown in Figure 10. It is observed that distance calculations are in a good agreement with real values.

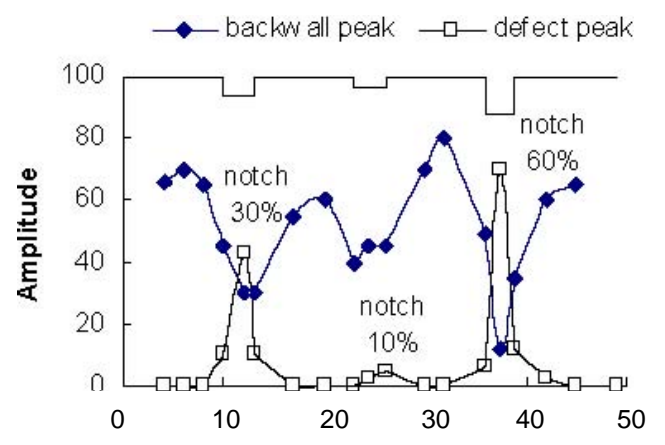

Distance across the width of plate, $\mathrm{cm}$

Fig. 8: The change of Amplitude of al mode, as scanned plate width (defect to probe distance was $75 \mathrm{~mm}$ ).

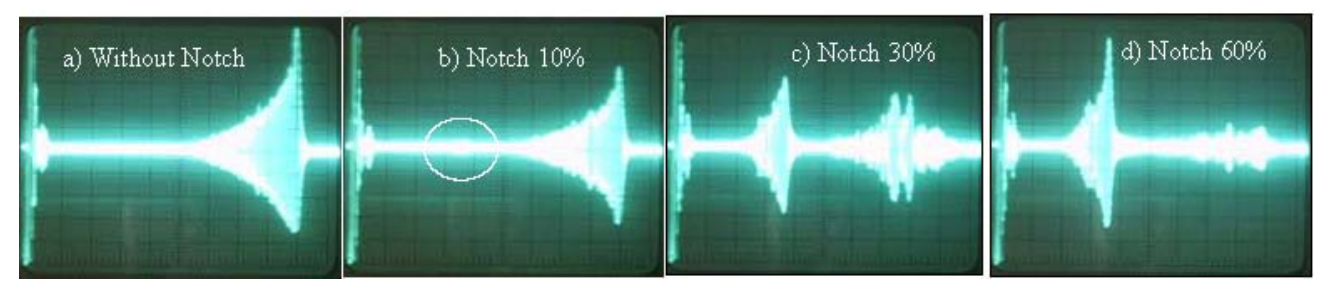

Fig. 9: The waveforms of ultrasonic lamb wave in pulse-echo technique, a1 mode evaluated (defect to probe distance was $75 \mathrm{~mm}$ ).

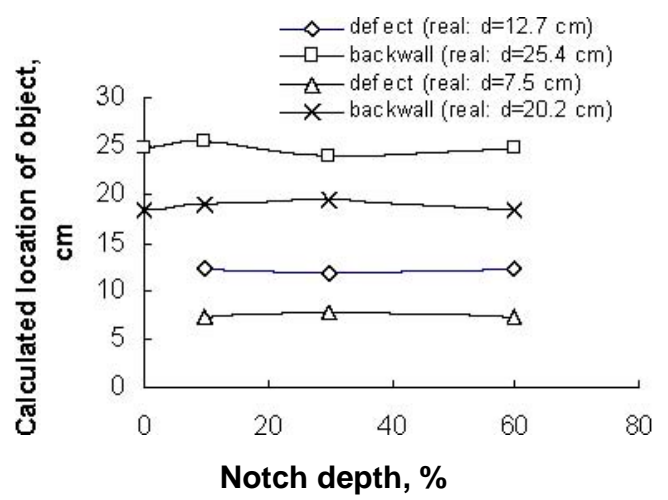

Fig. 10: The result of calculated location of object as compared with real values.

The waveforms of emission method have been shown in Figure 11, indicating that receiver peak will become weaker when a defected zone is scanned. Also, it reveals that increased depth of defects resulting in a more decreased of amplitude. In the emission method defect location can not be determined directly by analysis the waveform. But location of defect may be determined practically by connecting an Acrylic specimen in the beam direction as damper block. Amplitude decreases by connecting the damper block but in a defected zone higher decreased is occurred in 
comparison with the flawless areas. The location of defects can be found with an acceptable precision by this method.

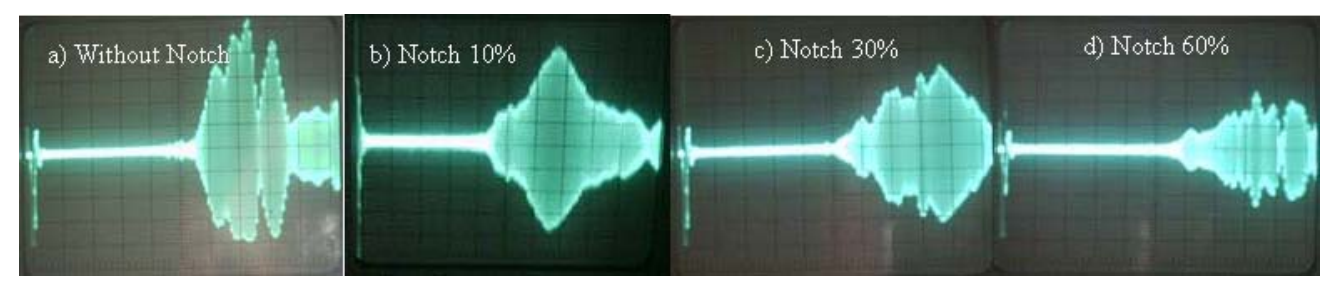

Figu. 11: The waveforms of ultrasonic lamb wave in emission technique, s1 mode evaluated (defect to transmitter distance and defect to receiver distance was $50 \mathrm{~mm}$ ).
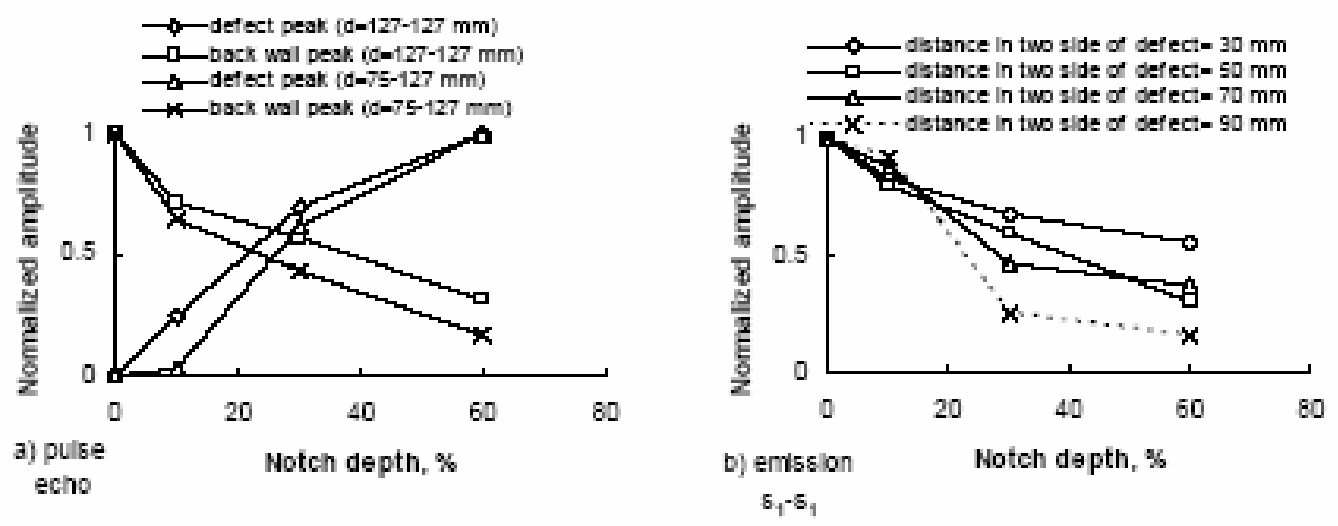

Fig. 12: The variations of normalized amplitude values as a function of defect depth in: a) pulseecho method and b) emission technique.

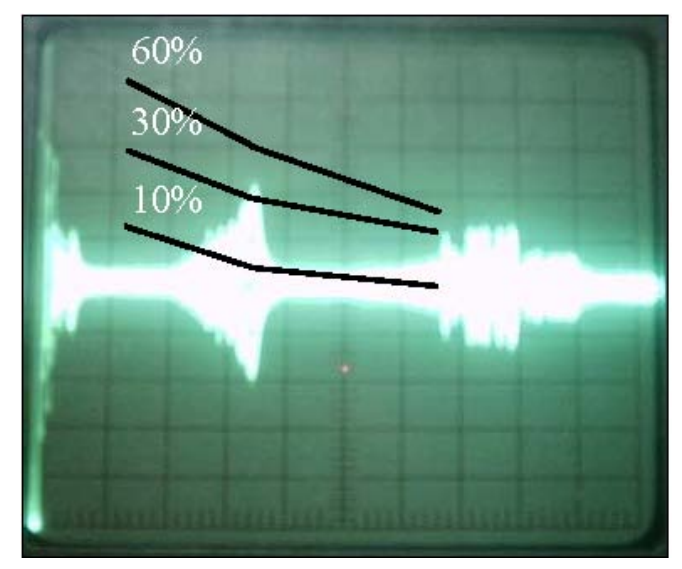

Fig. 13: The Drawing the DAC curve for defect evaluating by assessment the notch defects with the depth of $10 \%, 30 \%$ and $60 \%$ as reference defects.

Defect sizing is more difficult in ultrasonic lamb wave testing, due to its dispersive nature. In thin plates happily, it is usually important to flaw detecting and defect sizing isn't necessary in many cases. In lamb wave testing, defect length may be measured similar that one in bulk wave ultrasonic flaw detecting ( $6 \mathrm{db}$ drop low). This means that the start and end points of a defect can be calculated where the peak intensity of defect shows a $6 \mathrm{db}$ decrease. But height and depth of defects, detected by lamb wave, can not be measured by the common defect sizing methods. Amplitude in ultrasonic lamb wave testing gives some qualitative information about defect depth. The variations of amplitude as a function of defect depth, when using different ultrasonic 
lamb wave testing techniques, have been shown in Figure 12. It seems that in pulse echo method, the change of amplitude is more sensitive to defect depth than two other techniques.

We used a notch $10 \%$ as a reference for testing. A Distance-Amplitude-Correction curve (DAC) was made for evaluation. This diagram, as shown in Fig. 13, has three curves relating to notch $10 \%, 30 \%$ and $60 \%$. The observed signal in Fig. 13 related to a notch with $60 \%$ depth, which can be evaluated with good precision by drawing the DAC. But the amplitude analysis wasn't sometime repeatable. Experiments showed that notch defects with the depth of $10 \%$ and higher could be evaluated by used methods. Inspection was more difficult when probe to defect distance increased, due to attenuation, dispersion, beam speared effects and increased pulse duration. For long range inspections, low frequency probes should be used. These methods were fast and a large area of plates was inspected in a short time.

\section{Conclusions}

In the present work pulse-echo and emission lamb wave testing methods were used for interpretation of notch defects (10-60\% of through wall depth) and s1 and al modes were considered for inspections. It was found that these techniques could detect all fabricated defects. Inspection was more difficult as the probe to defect distance increased. Amplitude analysis showed that defect depth could be evaluated by drawing the DAC curves. Pulse echo was more sensitive for defect detection than emission technique. Location of defects was found by assessment the group velocity in pulse echo technique and the length of defect was determined by the law of $6 \mathrm{db}$ drop.

\section{References}

[1] T. Ghosh, T. Kundu and P. Karpur, 1998, "Efficient use of lamb modes for detecting defects in large plates", Ultrasonics, 36, pp. 791-801.

[2] T. Kundu, C. Potel and J. F. Belleval, 2001, "Importance of the near lamb mode imaging of multilayered composite plates", Ultrasonics, 39, pp. 283-290.

[3] J. L. Rose, D. Jiao and J. Spanner, 1996, "Ultrasonic guided wave NDE for piping, materials evaluation", Materials Evaluation, 54, pp. 1310-1313.

[4] J. L. Rose and M. J. Avioli, October 15-21 2000, "Elastic wave analysis for broken rail detection", Proceedings of 15 th World Conference on Non-Destructive Testing, Rome, Italy.

[5] J. L. Rose and X. Zhao, 2001, "Flexural mode tuning for pipe elbow inspection", Materials Evaluation, 59, pp. 621-624.

[6] T. R. Hay, L. Wei and J. L. Rose, 2003, "Rapid inspection of composite skin-honeycomb core structures with ultrasonic guided waves", Journal of Composite Materials, 37, pp. 929-939.

[7] D. E. Chimenti, 1997, "Guided waves in plates and their use in materials characterization", Applied Mechanics, 50, pp. 247-284.

[8] J. L. Rose,M. J. Quarry, A. V. Bray and C. J. Corley, October 20-24 1997, "Guided waves for corrosion detection potential in piping under insulation", Proceedings of ASNT Fall Conference, Pittsburgh, PA,USA.

[9] E. Pan, J. Rogers, S. K. Datta and A. H. Shah, 1999, "Mode selection of guided waves for ultrasonic inspection of gas pipelines with thick coating", Mechanics of Materials, 31, pp. 165-174.

[10] J. L. Rose, 2003, "Guided wave testing of water loaded structures", Materials Evaluation, 61, pp. 23-24. 
[11] J. L. Rose, "Dispersion curves in guided wave testing", Materials Evaluation, 61, pp. 2022.

[12] J. L. Rose, S. Pelts and M. Quarry, 1998, "A Comb transducer model for guided wave NDE", Ultrasonics, 36, pp. 163-168.

[13] D. P. Jansen and D. A. Hutchins, 1992, "Immersion tomography using Raleigh and lamb waves", Ultrasonics, 30, pp. 245-254.

[14] A. K. Kromine, P. A. Fomitchov, S. Krishnaswamy and J. D. Achenbach, 2000, "Laser ultrasonic detection of surface breaking discontinuities: scanning laser source technique", Materials Evaluation, 58, pp. 173-177.

[15] C. A. Paget, S. Grondel, K. Levin and C. Delebarre, 2003, "Damage assessment in composites by lamb waves and wavelet coefficients", Smart Materials and Structures, pp. 393-402.

[16] D. Alleyne and P. Cawley, 1991, "A two-dimensional Fourier transform method for the measurement of propagating multimode signals", Journal of Acoustic Society of America, 89, pp. 1159-1168.

[17] K. F. Graff, 1991, Wave motion in elastic solids, Dover Publication, NY, USA.

[18] P. D. Wilcox, 1998, "Lamb wave inspection of large structures using permanently attached transducers", Ph.D. Thesis, Imperial College of Science, Technology and Medicine.

[19] D. Levesque and L. Piche, 1992, "A robust transfer matrix formulation for the ultrasonic response of multilayered absorbing media", Journal of Acoustic Society of America, 92, pp. 452-467.

[20] E. L. Adler, 1990, "Matrix methods applied to acoustic waves in multilayers", IEEE Transaction on Ultrasonic Ferroelectric Frequency Control, 37, pp. 485-490.

[21] E. Pan, S. K. Datta, 1999, "Ultrasonic waves in multilayered superconducting plates", Journal of Applied Physics, 86, pp. 543-551.

\section{Appendix 1}

The followed equation can be used for predict the lamb wave phase velocity $\left(C_{P}\right)$ in a plate:

For symmetric modes: $\frac{\tan (q d / 2)}{\tan (p d / 2)}=\frac{-4 k^{2} \cdot p q}{\left(q^{2}-k^{2}\right)^{2}}$

For antisymmetric modes: $\frac{\tan (q d / 2)}{\tan (p d / 2)}=\frac{\left(q^{2}-k^{2}\right)^{2}}{-4 k^{2} \cdot p q}$

Where $\mathrm{k}$ is wave number of lamb wave and is given by: $k=\frac{2 \pi f}{C_{p}}$

$\mathrm{p}$ and $\mathrm{q}$ are calculated by: $p=k^{2}-\left(\frac{2 \pi f}{C_{L}}\right)^{2}$ and $q=k^{2}-\left(\frac{2 \pi f}{C_{T}}\right)^{2}$

$\mathrm{f}=$ linear frequency of lamb wave; $\omega=$ angular frequency $=2 \pi f ; C_{L}=$ longitudinal ultrasonic bulk wave velocity; $C_{T}=$ shear ultrasonic bulk wave velocity; $d=$ thickness of plate

Group velocity $\left(\mathrm{C}_{\mathrm{G}}\right)$ predicts as followed: $C_{G}=C_{p}\left(1-\frac{1}{1-\frac{C_{p}}{\alpha}}\right): \alpha=f d \frac{d C_{p}}{d(f d)}$ 